\title{
A Faster Earley Parser
}

\author{
Philippe McLean \& R. Nigel Horspool \\ Dept. of Computer Science, University of Victoria \\ Victoria, BC, Canada V8W 3P6 \\ E-mail: pmcleanecsc.uvic.ca, nigelhecsc.uvic.ca
}

\begin{abstract}
We present a parsing technique which is a hybrid of Earley's method and the LR(k) methods. The new method retains the ability of Earley's method to parse using arbitrary context-free grammars. However, by using precomputed LR(k) sets of items, we obtain much faster recognition speeds while also reducing memory requirements.
\end{abstract}

\section{Introduction}

The parsing method invented by Earley $[2,4]$ is a highly practical parsing technique for general context-free grammars (CFGs). If $n$ is the length of the input to be recognized, the parser requires time proportional to $n^{3}$ to recognize arbitrary context-free languages, $n^{2}$ for unambiguous languages, and $n$ for a large class of languages.

The amount of processing performed while recognizing an input string is large compared to table-driven techniques such as the LR parser family, which includes the LR(0), SLR(1), LALR(1) and LR(1) methods. These LR methods, however, cannot accept arbitrary CFGs. They are limited to subsets of unambiguous grammars. In general, the LR parsing table constructed for an arbitrary CFG will contain conflicts. That is, one or more states will provide a choice of actions to perform for some inputs.

A parsing method due to Tomita [6,4] overcomes the limitations of the LR methods. It uses LR tables that may contain conflicts. Whenever the parser encounters a choice of parsing actions, it in effect clones new copies of itself to track each of the conflicting actions simultaneously. Some copies of the parser may subsequently reach a state where parsing cannot proceed (i.e. the input symbol is invalid for that state) and these copies of the parsers simply terminate execution. In practice, the Tomita parser simulates parallel execution of multiple copies of a LR parser, and it uses a DAG data structure to reduce the storage needed by all the parse stacks. A Tomita parser is particularly efficient when few conflicts are encountered in the LR states.

If all we need to do is recognize the input, a Tomita parser would likely be the method of choice. However, we will usually wish to execute semantic actions while precisely one of the parses is being performed. This is not so easy for a Tomita parser because many parses are being performed in parallel. One possible solution is for each copy of the LR parser to construct a parse tree. At the end of the input, we can traverse one of these parse trees to perform the desired semantic actions. We consider that the 
computational work of building the parse trees negates the advantage of Tomita's method.

The Earley parser builds a data structure, a threaded sequence of states, which represents all possible parses of the input. After the input has been processed, it is straightforward to traverse the sequence of states to build a parse tree for one possible parse of the input, or to execute semantic actions for just the one parse.

We have developed a variation on Earley's method which, like Tomita's method, uses LR parse tables for efficiency, while retaining the advantage of permitting semantic actions to be easily associated with the grammar. The LR tables, in effect, capture precomputations of all the run-time actions performed by an Earley parser. Our parsing method, which we call LRE $(k)$, uses information from the LR tables and therefore avoids recomputing this information at run-time. The name $\operatorname{LEE}(k)$ reflects the fact that our method can be viewed as a combination of $L R(k)$ parsing with Earley parsing.

\section{Terminology and Notation}

\subsection{Context-Free Grammars}

A context free grammar $G$ is a four-tuple $\left\langle V_{T}, V_{N}, P, S t a r t\right\rangle$ where $V_{T}$ is a set of terminal symbols, $V_{N}$ is a set of nonterminal symbols, $V_{N} \cap V_{T}=\varnothing, P$ is a set of productions, and Start $\in V_{N}$ is the start symbol or goal symbol of the grammar. The vocabulary $V=V_{N} \cup V_{T}$.

An augmented grammar $G^{\prime}$ is formed from $\mathrm{G}$ by adding a special goal rule

$$
G^{\prime}=\left\langle V_{T} \cup\{-1\}, V_{N} \cup\left\{S^{\prime}\right\}, P \cup\left\{\text { Start }^{\prime} \rightarrow \vdash \text { Start }-1\right\} \text {, Start }{ }^{\prime}\right\rangle .
$$

where the tokens $\vdash$ and $f$ are delimiters that represent the beginning and end of input.

Lower-case letters near the front of the alphabet (i.e. $a, b, c$...) represent elements of $V_{T}$, upper-case letters near the front of the alphabet (i.e. A, B, C ...) represent elements of $V_{N}$, and upper-case letters near the end of the alphabet (i.e. $\mathrm{X}, \mathrm{Y}, \mathrm{Z}$ ) represent elements of $V$. A superscript represents repetitions of a symbol, so that, for example, $a^{3}$ represents the string aaa. Greek letters $\alpha, \beta, \ldots$ represent sequences of zero or more vocabulary symbols.

\section{$2.2 \quad$ LR $(k)$ Recognizers}

An item is a production which contains a marker, written as a dot, to indicate how much of the right-hand side (RHS) has been recognized. Associated with each item is a string of $k$ symbols $(k \geq 0)$. The string represents lookahead or right context for the production. For example, if $k$ is 2 , a possible item is [ $A \rightarrow a b \cdot B c, d d$ ]. This item indicates that we have matched the first two symbols on the right-hand side of the rule $A \rightarrow a b B$. If the complete RHS is successfully matched, then the next two symbols in the input should be dd for this production to be valid in a parse of the input at this point.

We use $S$ to denote the set of $\operatorname{LR}(k)$ sets of items for the augmented grammar $G^{\prime}$. Each element of $S$ corresponds to a state in the $\operatorname{LR}(k)$ recognizer for $\mathrm{G}^{\prime}$. The recognizer bas an initial state 


$$
\mathrm{I}_{\text {initial }}=\left\{\left[\text { Start }^{\prime} \rightarrow \cdot \vdash \text { Start }-\jmath^{-}-^{\mathbf{k}}\right]\right\} \in S,
$$

and it has an accept state

$$
I_{\text {accept }}=\left\{\left[\text { Start } \rightarrow \vdash \text { Start }-\dashv^{\prime}, \dashv^{k}\right]\right\} \in S .
$$

The transition function between the recognizer's states is

$$
\text { goto : } \mathrm{S} \times \mathrm{V} \rightarrow \mathrm{S} \cup\{\varnothing\}
$$

The function $\operatorname{goto}(I, x)$ is defined as the set of all items $\left[A \rightarrow \alpha x \cdot \beta, t_{1} \ldots t_{k}\right]$ such that $\left[A \rightarrow \alpha \cdot \times \beta, t_{1} \ldots t_{k}\right] \in I$. If the set $g o t o(I, x)$ is an empty set, the transition is illegal. (I.e., the string $\times t_{1} \ldots t_{k}$ cannot follow the symbols that have been accepted so far in a syntactically valid input.)

The closure of an itemset $I$ is defined as the least set $\mathrm{J}$ such that $\mathrm{I} \subseteq \mathrm{J}$, and $\left[A \rightarrow \alpha \cdot B \beta, t_{1} \ldots t_{k}\right] \in J$ implies that $\forall \eta\left(\eta \in \operatorname{first}_{k}\left(\beta, t_{1} \ldots t_{k}\right)\right):\{[B \rightarrow \bullet \gamma, \eta] \mid$ $B \rightarrow \gamma \in P\} \subseteq J$.

The function first $t_{k}(\beta, \gamma) \equiv_{\text {def }}\left\{\operatorname{prefix}_{k}(\sigma) \mid \beta \gamma \Rightarrow^{*} \sigma, \sigma \in V_{\mathrm{T}}^{*}\right\}$, where $\operatorname{prefix}_{\mathrm{k}}(\sigma)$ is the $k$-symbol prefix of $\sigma$.

The set of items for each state may be partitioned into kernel items and non-kernel items. The former are those items which are not added to a state by closure, while the latter (also called completion items) are those which are added to a state by closure.

\section{Conventional Earley Recognizers}

A conventional Earley recognizer has two inputs: a context-free grammar $G$ and a token string $x_{1} x_{2} \ldots x_{n}$, and determines if the string may be derived by G. For simplicity, lookahead will not be considered in this discussion $(k=0)$.

The recognizer constructs a sequence $E_{1}, E_{2} \ldots, E_{n+1}$, of sets of tuples. Each tuple has the form $<\mathrm{i}, \mathrm{p}>$ where $i$ is an item $[A \rightarrow \alpha \cdot \beta]$ and $p$ is an integer referring to the parent Earley set $E_{\mathrm{p}}$ where the tuple containing the item with the marker at the beginning of the RHS was introduced. The $k$-th set is formed as a result of recognizing the first $k$ - 1 input tokens.

Tuples in a state may be partitioned into active and predicted tuples. Active tuples may be introduced in two ways: by a SCANNER operation, and by a COMPLETER operation. The SCANNER operation introduces tuples from the previous state where the marker appears before the current input token; the marker is advanced past that token in the new item. This is the process of matching terminal tokens in a production's RHS, and corresponds to a shift operation in an LR parser. The COMPLETER operation identifies each tuple where an item's marker is at the end of a RHS, and moves the marker past the LHS in items in the tuple's parent state. This operation identifies the derivation of a non-terminal, in the recognition of some RHS; an LR parser would perform a reduction in exactly this case.

The COMPLETER operation introduces new tuples for every item where the marker appears before a non-terminal. This operation begins the recognition of possible derivations for a non-terminal; it is the closure of a set of items. Closure is performed at parse time in a conventional Earley parser. However these closure items are implicit in the $\mathrm{LR}(k)$ recognizer. 
Earley's doctoral dissertation [3] contains a proof of correctness for a conventional Earley recognizer, and an analysis of its algorithmic complexity. Parse trees may be enumerated for all derivations of the input string by examining the sets $\mathrm{E}_{\mathrm{i}}, 1 \leq i \leq n+1$.

The conventional recognizer affords a simple implementation. However, observation of the parser's actions reveals that the parser spends much of its time introducing new items during the completion operation. Many prediction items may not be used during the parse. The computation of item-set closures, a grammar-dependent operation, is performed at parse time. It is natural to wonder whether the Earley items can be grouped in a manner that exploits pre-computed properties of the grammar. Our solution is to group items into sets in exactly the same way as in the states of a deterministic (and possibly inadequate) LR( $k$ ) finite-state automaton.

\section{LRE - A Faster Earley Recognizer}

The new parsing method is named LRE $(k)$; this represents the hybrid nature of the algorithm as a composition of the $L R(k)$ and Earley parsing methods.

In the following description, we use $x_{1} x_{2} \ldots x_{n}$ to represent the input to the recognizer. So that lookahead sets are properly defined, we assume that the input is terminated by $k$ end-of-file delimiters. I.e., $x_{n+i}=-1$, for $1 \leq i \leq k$.

Our algorithm is based on a conventional Earley parser and its correct operation may be established by comparing its actions to an Earley parser's actions. A conventional Earley parser uses items of the form $\left[A \rightarrow \alpha \cdot \beta, t_{1} \ldots t_{k}, p\right]$, where $A \rightarrow \alpha \cdot \beta$ is a marked production, $t_{1} \ldots t_{k}$ is the lookahead for the item, and $p$ is a reference back to the state where recognition of the rule $A \rightarrow \alpha \beta$ commenced. Our algorithm takes advantage of the fact that the first two components of the Earley item represent an item in one or more states of the $\operatorname{LR}(k)$ recognizer. We therefore implement states in our LRE parser in terms of states in the LR( $k)$ recognizer. The advantages of our representation are (1) we can use the $\operatorname{LR}(k)$ recognizer's tables to determine actions for the Earley parser, (2) the lookahead strings are not computed dynamically, and (3) the new representation can be implemented in a manner which uses much less storage.

A state in our LRE recognizer will be called an Earley state, and will be written as $E_{m}$. State $E_{m}$ is reached after recognizing the token string $x_{1} x_{2} \ldots x_{m-1}$. The state $E_{m}$ is represented by a set of tuples $\left\{\left(\mathrm{I}_{1}, \mathrm{~B}_{1}\right),\left\langle\mathrm{I}_{2}, \mathrm{~B}_{2}\right), \ldots\right\}$ where each $\mathrm{I}_{\mathrm{i}} \in S$ is the number of some state in the $L R(k)$ recognizer and $B_{i}$ is an organized collection of back-pointers to Earley states. In programming terms, each $B_{j}$ could be implemented as an array of lists of LRE state numbers, where elements in the array are in one-to-one correspondence with items in $L R(k)$ state $I_{i}$. In more formal terms, we can represent $B_{i}$ as a list of list of integers $\left[\left[b_{i 11}, b_{i 12}, b_{i 1}, \ldots\right],\left[b_{i 21}, b_{i 22}, \ldots\right], \ldots\left[b_{i n}, b_{i n}, \ldots\right]\right]$ where each $b_{i x} y$ is an integer in the range 0 to $k$ inclusive, and $\operatorname{LR}(k)$ state $I$ has $n$ items.

As an example, suppose that $L R E$ state $E_{3}$ has the following representation:

$$
\{\langle 17,[[1,2],[3],[3]]),\langle 23,[[2]]\rangle\}
$$

This would mean that state $E_{3}$ represents a mixture of the same items as found in the LR( $k$ ) states numbered 17 and 23. State 17 must have three items (the length of the list that completes the tuple with state number 17) - let us suppose that these items are: 


$$
\begin{array}{ll}
A \rightarrow A \cdot B C & \alpha_{1} \\
X \rightarrow a A \cdot D & \alpha_{2} \\
A \rightarrow \cdot b & \alpha_{3}
\end{array}
$$

where we have written the lookahead strings as $\alpha_{1}, \alpha_{2}$ and $\alpha_{3}$ respectively. Similarly, LR( $k$ ) state 23 must have just one item and let us suppose that this item is

$$
C \rightarrow a b \cdot b \quad \beta_{1}
$$

Now, our LRE state represents an Earley state which contains exactly these items:

$$
\begin{aligned}
\left\{\left(A \rightarrow A \cdot B C, \alpha_{1}, 1\right\rangle,\left\langle A \rightarrow A \cdot B C, \alpha_{1}, 2\right\rangle,\left\langle X \rightarrow a A \cdot D, \alpha_{2}, 3\right\rangle,\right. & \\
\left.\left\langle A \rightarrow \cdot b, \alpha_{3}, 3\right\rangle,\left\langle C \rightarrow a b \cdot b, \beta_{1}, 2\right\rangle\right\} &
\end{aligned}
$$

The first tuple in $E_{3}$ represents two copies of the first item of LR state 17, where one copy is associated with a pointer back to state 1 and the other with a pointer back to state 2. And similarly for the other items in LR states 17 and 23.

Our parsing algorithm is based on Earley's, but it has been modified to work with the different state representation. It has two main functions named SCAN and RECOGNIZER.

Given a LRE state $E_{s}$, the function $\operatorname{SCAN}\left(E_{s}, X, t\right)$ constructs a new LRE state which represents Earley items where the marker has been past the token $X$ in all applicable Earley items represented in set $E_{s}$.

The procedure RECOGNIZER $\left(x_{1}, \ldots, x_{n}, \ldots, x_{n+k}\right)$ determines whether the token string $x_{1} \ldots x_{n}$ is in the language generated by $G$. Note that each of the symbols $x_{n+1}$, $\mathrm{x}_{\mathrm{n}+2} \ldots \mathrm{x}_{\mathrm{n}+\mathrm{k}}$ is the symbol - . These extra $k$ symbols are needed to provide right context for the final reductions in the parse. RECOGNIZER constructs a sequence of Earley states, from which a set of valid parse trees may be enumerated. Code for the SCAN function is shown in Figure 1, while code for the RECOGNIZER is given in Figure 2.

The code uses the data structures and tables explained below. The tables may be created during the $\operatorname{LR}(k)$ parser construction algorithm.

- Each LRE state is represented by a set whose elements are structures with two fields. One field is named state and holds a state number for the $L R(k)$ recognizer. The other field is named BackPtrs and is an array of lists of integers. An element BackPtrs [ $i$ ] holds the state numbers that should be associated with the $i$-th item of $\mathrm{LR}(k)$ state with number State.

- The array Numberof Items [i] gives the number of items in LR $(k)$ state $i$.

- The array SHIFT $[s, x]$ holds the shift actions for the $\operatorname{LR}(k)$ recognizer. If the current $\operatorname{LR}(k)$ state is numbered $s$, then $\operatorname{SHIFT}[s, \mathrm{x}]$ gives the number of the destination state to shift on symbol $x$. If a valid shift action is not defined for symbol $x$, $\operatorname{SHIFT}[s, \mathrm{x}]$ holds -1 .

- The array DestItemPosition [ $m, i]$ gives the correspondence between items in one $\operatorname{LR}(k)$ state and those in another $\operatorname{LR}(k)$ state. In particular, if item $i$ in the $\operatorname{LR}(k)$ state numbered $m$ is $A \rightarrow \alpha \cdot X \beta$, then a shift on the symbol $X$ will lead to a unique destination $\operatorname{LR}(k)$ state that contains the item $A \rightarrow \alpha X \cdot \beta$. The number held in Dest Itemposition [ $m, i]$ is the number of this item in the destination state. If item $i$ in state $m$ does not have the specified form (i.e. the marker is at the end of the right-hand side), we assume that Dest ItemPosition [m, i] holds the value -1 . 


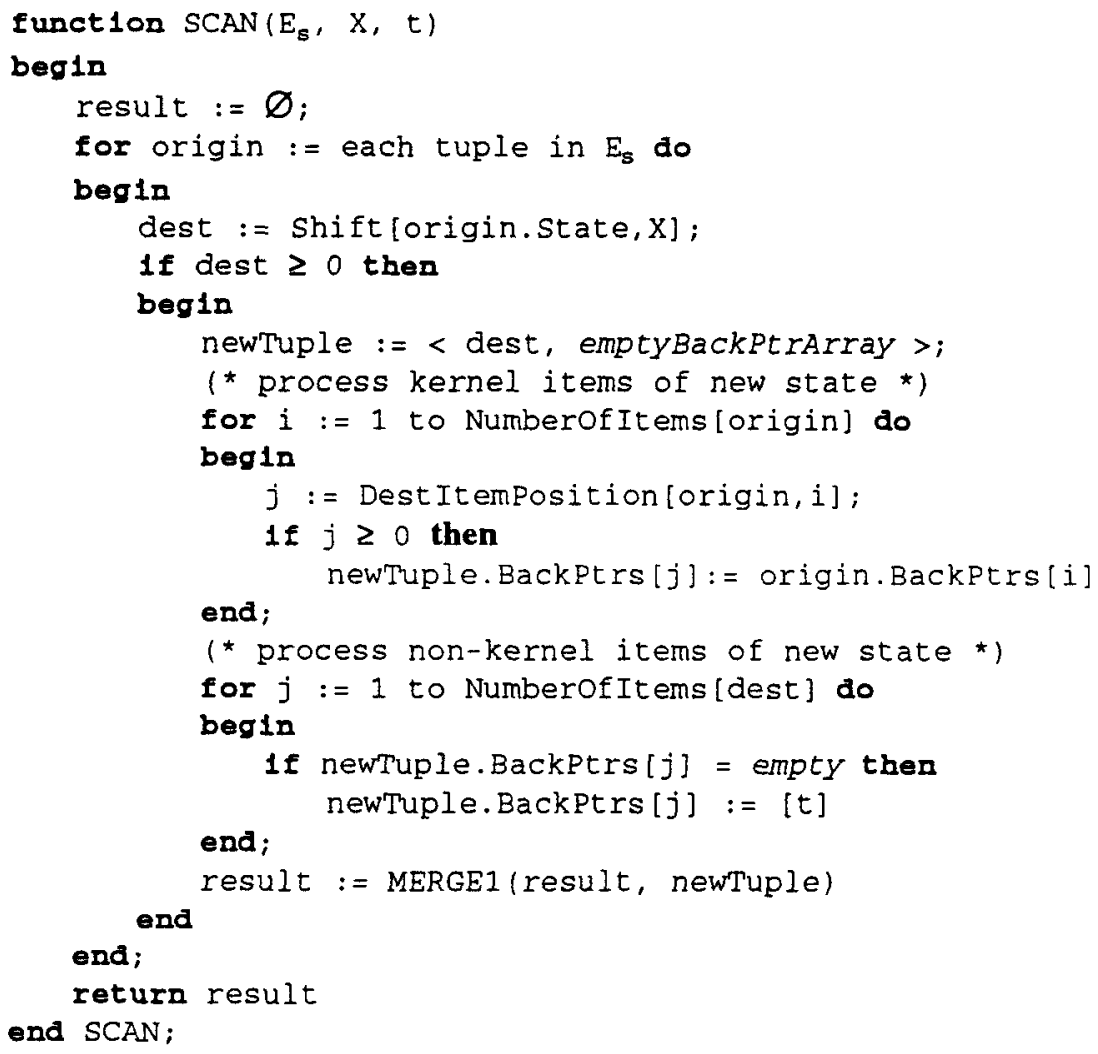

Fig. 1. The SCAN Function 


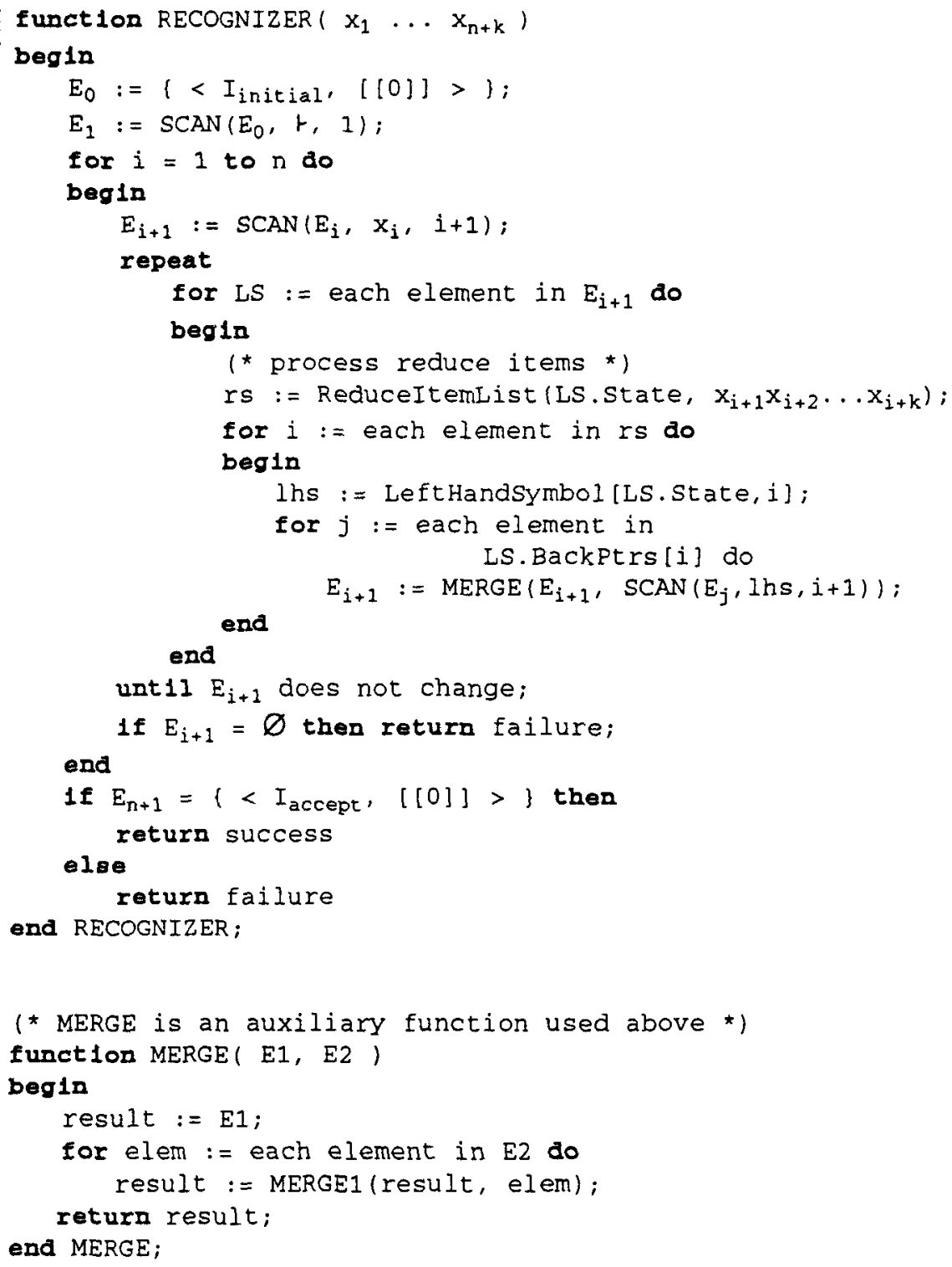

(* MERGE is an auxiliary function used above *) funct 1on MERGE( E1, E2)

begin

Fig. 2. The RECOGNZER Function 
- The array Reduce It emL ist $[m, \alpha]$ is a list of the positions of all items in $L R(k)$ state $m$ where the marker is at the end of the right-hand side and where the lookahead string for these items is $\alpha$.

- The array LeftHandSymbol $[m, i]$ gives the symbol which appears on the lefthand side of the $i$-th item in LR( $k)$ state $m$.

\section{An Example of Operation}

To illustrate the operation of the $\operatorname{LRE}(k)$ parsing method, we use the ambiguous grammar:

$$
\begin{aligned}
& \text { 1. } E \rightarrow E+E \\
& \text { 2. } E \rightarrow n
\end{aligned}
$$

This grammar is augmented by the extra rule

$$
0 \text {. } S \rightarrow+E-1
$$

For simplicity, we choose $k=0$. From this grammar, we can derive the $\mathrm{LR}(0)$ recognizer which has the states and actions shown below in Table 1. Each shift action is preceded by the symbol which selects that shift action. Because a $L R(0)$ parser does not use lookahead, a reduce action is performed no matter what the next symbol is. The word any represents the fact that any symbol selects the specified reduce action. The table contains conflicts, in particular note that state 7 implicitly contains two different

\begin{tabular}{|c|c|c|c|c|}
\hline State & $\begin{array}{l}\text { Item } \\
\text { No. }\end{array}$ & Item & \multicolumn{2}{|c|}{ Parse Actions } \\
\hline 1 & 1 & {$[S \rightarrow \bullet \vdash E f]$} & $r$ & Shift 2 \\
\hline \multirow{3}{*}{2} & 1 & {$[S \rightarrow \vdash \cdot E-1]$} & \multirow{3}{*}{$\begin{array}{l}E \\
n\end{array}$} & \multirow{3}{*}{$\begin{array}{l}\text { Shift } 3 \\
\text { Shift } 4\end{array}$} \\
\hline & 2 & {$[E \rightarrow \cdot E+E]$} & & \\
\hline & 3 & {$[E \rightarrow \cdot n]$} & & \\
\hline \multirow{2}{*}{3} & 1 & {$[S \rightarrow \vdash E \bullet-1]$} & \multirow{2}{*}{$\begin{array}{l}+ \\
-1\end{array}$} & \multirow{2}{*}{$\begin{array}{l}\text { Shift } 6 \\
\text { Shift } 5\end{array}$} \\
\hline & 2 & {$[E \rightarrow E \cdot+E]$} & & \\
\hline 4 & 1 & {$[E \rightarrow n \cdot]$} & any & Reduce 2 \\
\hline 5 & 1 & {$[S \rightarrow \vdash E+\bullet]$} & any & Reduce 0 \\
\hline \multirow{3}{*}{6} & 1 & {$[E \rightarrow E+\cdot E]$} & \multirow{3}{*}{$\begin{array}{l}E \\
n\end{array}$} & \multirow{3}{*}{$\begin{array}{l}\text { Shift } 7 \\
\text { Shift } 4\end{array}$} \\
\hline & 2 & {$[E \rightarrow \cdot E+E]$} & & \\
\hline & 3 & {$[E \rightarrow \cdot n]$} & & \\
\hline & 1 & {$[E \rightarrow E+E \cdot]$} & \multirow{2}{*}{$\begin{array}{l}+ \\
\text { any }\end{array}$} & \multirow{2}{*}{$\begin{array}{l}\text { Shift } 6 \\
\text { Reduce } 1\end{array}$} \\
\hline & 2 & {$[E \rightarrow E \bullet+E]$} & & \\
\hline
\end{tabular}
actions for the case when the lookabead symbol is + .

Table 1: LR(0) Recognizer for the Example Grammar 
From that $L R(0)$ table we derive the tables shown below in Figure 3. Only the significant entries in the two rectangular arrays, Destitemposition and LeftHandSymbol are shown (the missing elements in these arrays should never be accessed). Similarly, only the significant entries in the Shift array are shown; if any other element is accessed the result should be -1.

Fig. 3. Tables Used In Parser Example

\begin{tabular}{|c|c|c|c|}
\hline State & $\begin{array}{c}\text { Item } \\
\text { No. }\end{array}$ & $\begin{array}{c}\text { Dest Item } \\
\text { Position }\end{array}$ & $\begin{array}{c}\text { Left Hand } \\
\text { Symbol }\end{array}$ \\
\hline 1 & 1 & 1 & S \\
\hline 2 & 1 & 1 & S \\
\hline 2 & 2 & 2 & E \\
\hline 2 & 3 & 1 & E \\
\hline 3 & 1 & 1 & S \\
\hline 3 & 2 & 1 & E \\
\hline 4 & 1 & -1 & E \\
\hline 5 & 1 & -1 & S \\
\hline 6 & 1 & 1 & E \\
\hline 6 & 2 & 2 & $E$ \\
\hline 6 & 3 & 1 & $E$ \\
\hline 7 & 1 & -1 & $E$ \\
\hline 7 & 2 & 1 & $E$ \\
\hline
\end{tabular}

\begin{tabular}{|c|c|c|}
\hline State & $\begin{array}{c}\text { Number } \\
\text { Of } \\
\text { Items }\end{array}$ & $\begin{array}{c}\text { Reduce- } \\
\text { It temList }\end{array}$ \\
\hline 1 & 1 & {[]} \\
\hline 2 & 3 & {[]} \\
\hline 3 & 2 & {[]} \\
\hline 4 & 1 & {$[1]$} \\
\hline 5 & 1 & {$[1]$} \\
\hline 6 & 3 & {[]} \\
\hline 7 & 2 & {$[1]$} \\
\hline
\end{tabular}

\begin{tabular}{|c|c|c|}
\hline State & Symbol & Shift \\
\hline 1 & $f$ & 2 \\
\hline 2 & $E$ & 3 \\
\hline 2 & $n$ & 4 \\
\hline 3 & + & 6 \\
\hline 3 & + & 5 \\
\hline 6 & $E$ & 7 \\
\hline 6 & $n$ & 4 \\
\hline 7 & + & 6 \\
\hline
\end{tabular}

We now trace the states of the LRE(0) parser on the input string $n+n+n$. The RECOGNIZER function begins by initializing the set $E_{0}$ with the initial LRE state $\{\langle 1,[0]\rangle\}$. It represents item 1 of state 1 in the $\operatorname{LR}(0)$ recognizer - indicating that the RHS of the rule $S \rightarrow \vdash E-t$ is to be recognized.

Each numbered step in our trace corresponds to the processing of one input symbol, and begins by showing the LRE state that is computed after seeing that input symbol. An explanation of the state's derivation is provided for the first few steps only.

$==$ The start of input symbol $t$ is processed $=$

1. $\mathrm{E}_{1}=\{\langle 2,[[0],[1],[1]]\rangle\}$. RECOGNIZER called $\operatorname{SCAN}\left(\mathrm{E}_{0}, \vdash, 1\right)$, which looked 
up the action for LR( 0$)$ state 1 when the input is + . Thus it created the LRE item $\langle 2,[[],[],[]\rangle)$ and then it filled in the back pointers. The list [0] was copied from the origin item, while the two lists containing [1] correspond to completion items.

$==$ The first input symbol $\mathrm{n}$ is now processed $=$

2. $E_{2}=\{\langle 4,[[1]]\rangle,\langle 3,[[0],[1]]\rangle\}$. RECOGNIZER called $\operatorname{SCAN}\left(E_{1}, n, 2\right)$. The (4, [ [1] ] ) element is created because of the $\operatorname{LR}(0)$ action for state 2 when the lookahead symbol is $n$. The other items are created by RECOGNIZER because item 1 in LR(0) state 4 is a reduce item, and the reduce action is triggered by the next input symbol which is +. The LHS symbol for that item is E, and RECOGNIZER called $S C A N\left(E_{1}, E, 1\right)$ to create the two extra items.

$=$ The second input symbol + is processed $==$

3. $E_{3}=\{\langle 6,[[1],[3],[3]]\rangle\}$.

$==$ The input symbol $n$ is processed $=$

4. $E_{4}=\{\langle 4,[[3]]\rangle,\langle 7,[[1],[3]]\rangle,\langle 3,[[0],[1]]\rangle\}$.

$==$ The input symbol + is processed $==$

5. $E_{5}=\{\langle 6,[[1,3],[5],[5]]\rangle\}$.

$==$ The input symbol $n$ is processed $==$

6. $\mathrm{E}_{6}=\{\langle 4,[[5]]\rangle,\langle 7,[[1,3],[3,5]]\rangle,\langle 3,[[0],[1]]\rangle\}$.

$==$ The end-of-input symbol $t$ is processed $==$

7. $E_{7}=\{\langle 5,[0]\rangle\}$.

\section{An Additional Enhancement}

The algorithm presented above can be further improved. The implementation used in our experiments does not immediately record non-kernel items in a LRE state (except when handling productions with an empty RHS). Their processing is deferred until scanning to the next state occurs. By recording the number of kernel items in each LR( $k)$ state, and by consulting the Dest ItemPos ition table, it can be determined whether or not a particular item in a destination state came from a kernel item in the source state. If it did, the BackPtr list is copied from the previous state. If it did not, the list $[t-1]$ is supplied, where $t$ is the number of the current LRE state.

The additional improvement achieves significant space and time savings, because many predictions items in an Earley parser are fruitless

\section{Experimental Results}

Lookahead significantly affects the speed of an Earley parser. In general, it is used to eliminate items from the sets of items maintained by the parser. Fewer items imply that fewer fruitless parsing possibilities are explored. On the other hand, a conventional 
Earley parser computes the lookahead contexts for items at run-time, and choosing a large value for the lookahead $k$ will waste execution time. In Figure 4, we compare the speed of a conventional Earley parser and our LRE parsing method for $k=0$ and $k=1$.

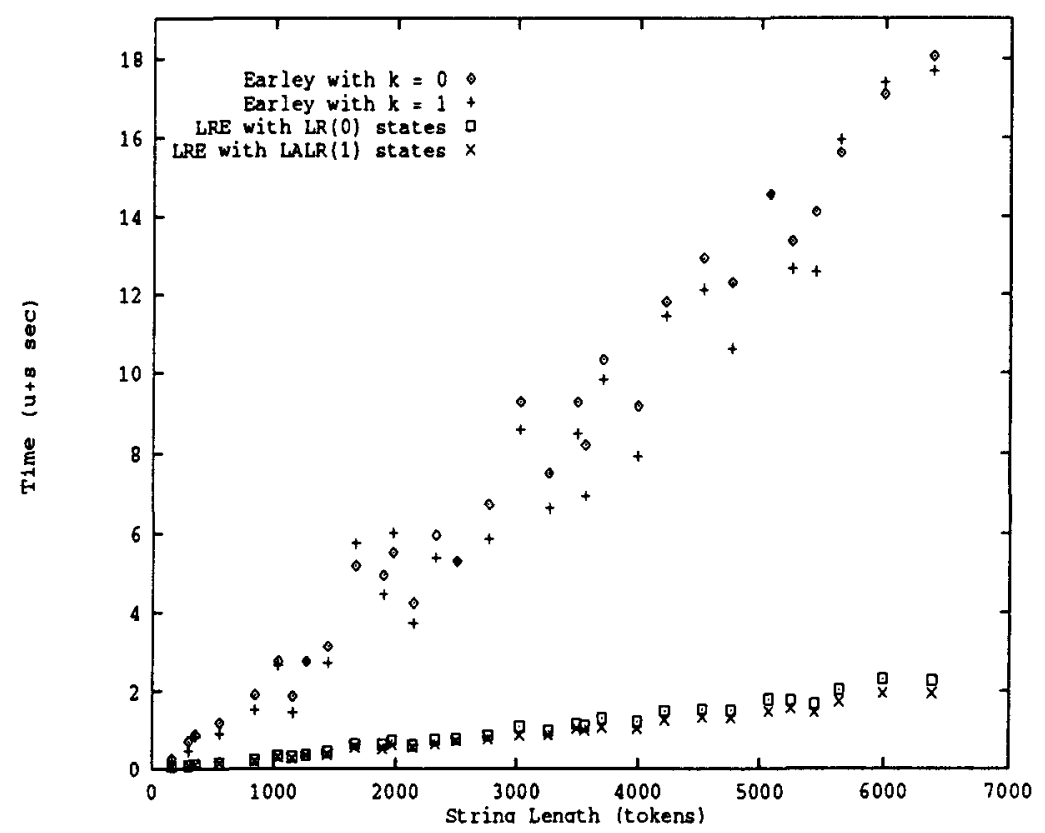

Fig. 4. Effect of Lookahead on Parsing Speed

Figure 4 already demonstrates that $\operatorname{LRE}(k)$ is a much faster parsing method than the conventional Earley parsing method. In Figure 5, we show an additional comparison against a parser generated by the freely distributed parser generator bison [1]. (Other measurements, not displayed here, reveal that a parser generated by yacc [5] yields very similar results.) Our grammar for these experiments was Roskind's ANSI C grammar. The grammar contains one ambiguity, namely the dangling else problem. This ambiguity is automatically eliminated from the generated parser when yacc and bison are used; it is retained by the Earley parsers.

For an unambiguous grammar (or when the ambiguities have been eliminated, such as with the bison's interpretation of the Roskind C grammar), recognition time is proportional to the length of the input. For an ambiguous grammar, the recognition time may increase as the cube of the length of the input. Figure 6 shows timing measurements when parsing with the ambiguous grammar:

$$
\mathrm{S} \rightarrow \mathrm{S} S \mathrm{I} \text {, }
$$



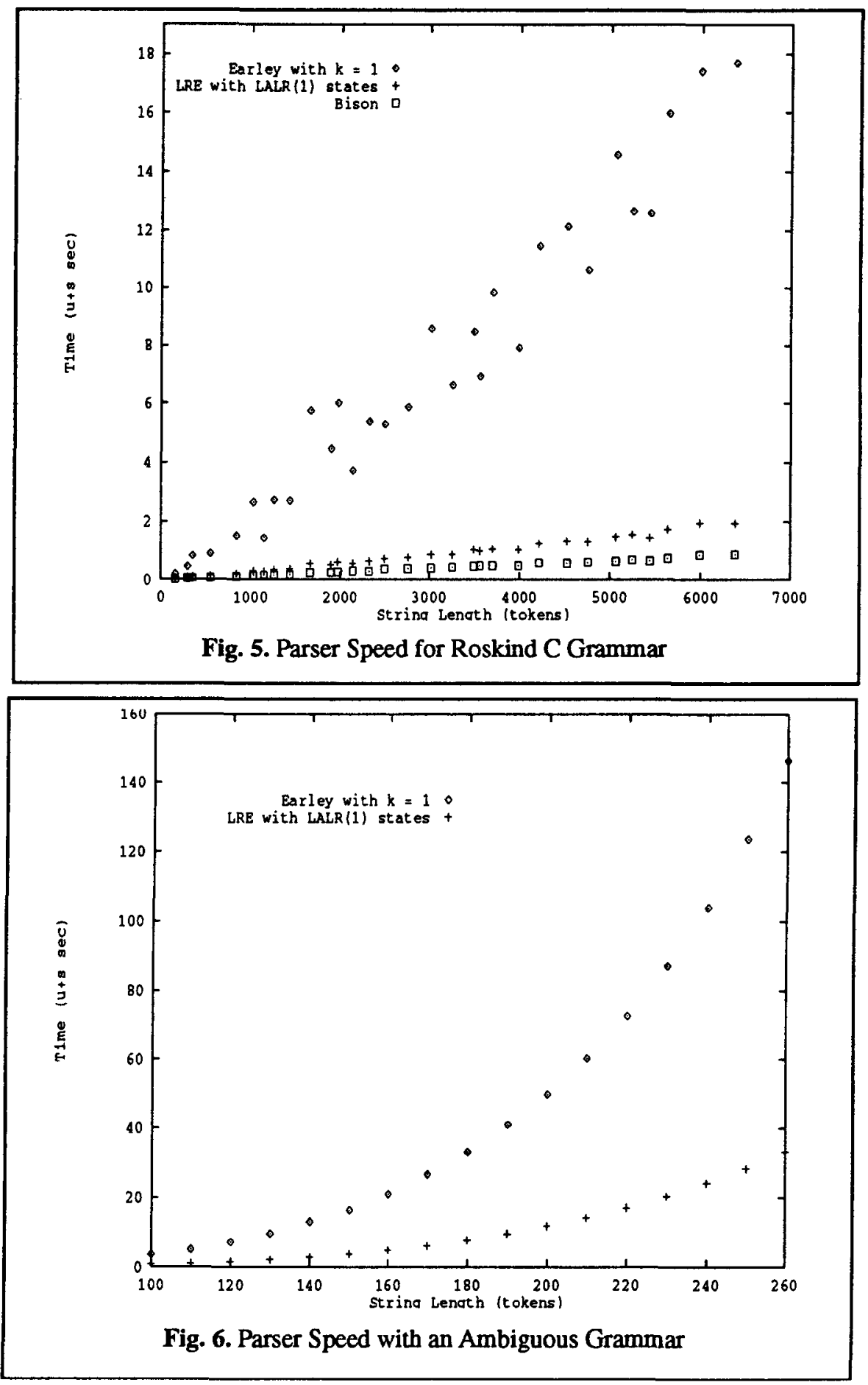


\section{Conclusions}

We have modified Earley's parsing method so that it can take advantage of precomputed $\operatorname{LR}(k)$ sets of items. The result is a hybrid parsing method, LRE $(k)$, which can still handle general context-free grammars but which is comparable in speed to a yaccgenerated or bison-generated parser. However, yacc and bison can, of course, only recognize unambiguous languages that are based on LALR(1) grammars with conflict elimination in the generated parser. The LRE $(k)$ parsing method is 10 to 15 times faster than a conventional Earley parser, while requiring less than half the storage.

\section{Acknowledgements}

Funding for this research was provided by the Natural Sciences and Engineering Research Council of Canada in the form of a summer fellowship for the first author and a research grant for the second author. The initial motivation for working on this problem is due to Gordon Cormack.

\section{References}

1. Donnelly, C., and Stallman, R. BISON: Reference Manual. Free Software Foundation, Cambridge, MA, 1992.

2. Earley, J. An Efficient Context-Free Parsing Algorithm. Comm. ACM 13, 2 (Feb. 1970), 94-102.

3. Earley, J. An Efficient Context-Free Parsing Algorithm. Ph.D. Thesis, CarnegieMellon University, 1968.

4. Grune, D., and Jacob, C.J.H. Parsing Techniques: a practical guide. Ellis Horwood, Chichester, 1990.

5. Johnson, S.C. YACC: Yet Another Compiler-Compiler. UNIX Programmer's Supplementary Documents, vol 1, 1986.

6. Tomita, M. Efficient Parsing for Natural Language. Kluwer Academic Publishers, Boston, 1986. 